\title{
Systemische Therapie, die Schweizerische Gesellschaft für systemische Therapie und Beratung und die «European Family Therapy Association» (EFTA)
}

\author{
O. Wäger, D. Krähenbühl, R. Traube, G. Fueter
}

Die Psychotherapie mit ihren verschiedenen Ausrichtungen ist aus der medizinischen Landschaft nicht mehr wegzudenken. Es besteht ein breiter Konsens darüber, dass sich die psychiatrisch-psychotherapeutische Versorgung am bio-psycho-sozialen Krankheitsmodell orientiert. Seelische Störungen manifestieren sich im biologischen Bereich, im persönlichen Erleben und im sozialen Verhalten und sind über diese drei Ebenen sowie deren Wechselwirkungen beeinflussbar. Hier setzt die systemische Therapie ein, indem sie die Muster, welche sich auf den Ebenen zeigen, deutlich werden lässt und mit den Beteiligten Veränderungsmöglichkeiten erarbeitet. Die Schweizerische Gesellschaft für systemische Therapie und Beratung hat es sich zur Aufgabe gemacht, systemisches Denken und Handeln in Theorie und Praxis zu fördern, deshalb wird sie hier porträtiert.

Korrespondenz:

Dr. med. Otmar Wäger

Seemattstrasse 2

CH-8180 Bülach

\section{Einleitung}

Systemische Therapie ist ein eigenständiges psychotherapeutisches Verfahren mit einer Vielzahl von Methoden und Anwendungsbereichen. Ursprünglich aus der Familientherapie einzelnen, Paaren, Familien und Gruppen angewandt. Sie betrachtet die Patientinnen und Patienten im Beziehungsgefüge ihres Umfeldes und sieht Symptome im Zusammenhang mit bestimmten Beziehungsmustern. Therapeutische Interventionen zielen darauf $\mathrm{ab}$, diese Muster deutlich werden $\mathrm{zu}$ lassen und zu verändern, die Ressourcen zu aktivieren und hilfreiche Handlungsmöglichkeiten der Beteiligten zu erweitern. Deshalb werden wo möglich und erwünscht Bezugspersonen des Patienten beziehungsweise der Patientin, die bereit sind, an Lösungen mitzuarbeiten, in den Therapieprozess einbezogen. Die Ausgestaltung des Therapiesettings richtet sich nach den Erfordernissen einer für alle Beteiligten befriedigenden Problemlösung. Systemisch nennen wir alles therapeutische Denken und Handeln, das sich einer Mehrpersonenperspektive und zirkulären Kausalvorstellungen öffnet. Eine systemische Sicht geht davon aus, dass zwischen biologischer, psychischer und sozialer Ebene dynamische entwickelt, wird sie heute in der Arbeit mit
Wechselwirkungen stattfinden. Die Wirkungen beispielsweise einer Psychose oder einer Anorexie sind, systemisch gesehen, nicht auf das Individuum beschränkt, sondern greifen in dessen Umfeld hinein - und zurück. Dadurch entstehen signifikante und dem Individuum übergeordnete Wirkmuster, die dessen Leiden unterhalten und verstärken können. Systemische Hilfe integriert daher all jene Personen, die in eine Krankheitsdynamik einbezogen und an den Lösungen interessiert sind, in einem sogenannten «therapeutischen System». Systemische Therapie nutzt die wissenschaftlich gut abgesicherte «Heilkraft» zielmotivierter Vertrauensbeziehungen zwischen Patientinnen und Patienten und nahen Bezugspersonen.

Im Weiterbildungsprogramm zum Facharzt für Psychiatrie und Psychotherapie der FMH zählt die systemische Therapie nebst der analytischen und kognitiv-verhaltensorientierten Therapie zu den drei anerkannten Modellen. Systemische Therapie bzw. ihre Methodik eignet sich gut zur Kombination mit anderen Therapiemethoden, z.B. der kognitiv-verhaltenstherapeutischen.

Systemische Therapie sieht sich den Standards der «Evidence-based Medicine» (EBM) verpflichtet, was das wirksamste, belastungs- und risikoärmste Vorgehen betrifft.

\section{Schweizerische Gesellschaft für systemische Therapie und Beratung (SGS)}

Die Schweizerische Gesellschaft für systemische Therapie und Beratung wurde 1989 mit dem Ziel der Weiterentwicklung und Verbreitung der systemischen Denk- und Arbeitsweise in der Schweiz gegründet. Der Gesellschaft gehören Mitglieder aus den drei Berufsbereichen Medizin, Psychologie und Sozialarbeit an. 


\section{Ziele der SGS}

$\mathrm{Zu}$ den Zielsetzungen der SGS gehört die Förderung einer institutionsübergreifenden, zielorientierten systemischen Therapie und Beratung in ihrer praktischen Anwendung, einer qualitativ hochstehenden Ausbildung in systemischer Therapie und Beratung, die Weiterbildung von praktizierenden systemischen Therapeuten/-innen und systemischen Beratern/-innen, die Forschungstätigkeit auf dem Gebiet der systemischen Therapie und Beratung, die Öffentlichkeitsarbeit bezüglich der systemischen Denk- und Handlungsweisen sowie die Zusammenarbeit mit Organisationen von verwandten Zielsetzungen.

Mitglieder der SGS erarbeiten derzeit zusammen mit Mitgliedern anderer systemorientierter Gruppierungen Strukturen für einen Dachverband aller in der Schweiz tätigen systemtherapeutischen Gesellschaften.

\section{Aktivitäten der SGS}

Die Mitglieder treffen sich in interdisziplinären Intervisions- und Supervisionsgruppen bzw. Qualitätszirkeln. Die Gruppen sind auf die Agglomerationszentren und Regionen verteilt.

Alljährlich finden zwei Mitgliederversammlungen mit Vorträgen und Workshops statt.

In verschiedenen Kommissionen und Arbeitsgruppen der SGS werden aktuelle Themen bearbeitet. So wurden Kriterien für eine fundierte systemtherapeutische Ausbildung geschaffen. Die Ausbildungsangebote werden in einem kontinuierlichen Prozess gesichtet und bewertet. Richtlinien für den juristisch korrekten Einsatz von audiovisuellen Mitteln in der Therapie sind zusammengetragen worden. Für die Vereinsmitglieder bestehen verbindliche Ethikrichtlinien. 1999 beauftragte die SGS zwei externe Forschungsfachleute mit der Durchführung einer Multicenterevaluationsstudie zur Ergebnisqualität ambulanter systemischer Therapie. Die Studie, welche sich auf die Datenerhebung im Feld stützt und somit eine Überprüfung der Wirksamkeit in der Praxisrealität darstellt, konnte im Frühjahr 2001 erfolgreich abgeschlossen werden. Die Ergebnisse wurden in der Zeitschrift «Psychiatrie, Psychotherapie und medizinische Psychologie» (Hrsg. Franz Caspar) publiziert und sind unter www.systemis.ch sowie www.orgalife.ch abrufbar.

\section{Ärztesektion der SGS (AS-SGS)}

Die Ärztesektion der SGS ist angegliederte Gesellschaft der Schweizerischen Gesellschaft für Psychiatrie und Psychotherapie (SGPP). Unter anderem über Vorstandsmitglieder und
Delegierte der kantonalen Fachgesellschaften ist die SGS eng mit der SGPP und mit der Schweizerischen Gesellschaft für Kinder- und Jugendpsychiatrie und -psychotherapie (SGKJPP) vernetzt.

In einer Arbeitsgruppe erarbeitete die AS-SGS Ausbildungsanforderungen in systemischer Therapie für Ärztinnen und Ärzte in Weiterbildung zur Fachärztin/zum Facharzt FMH für Psychiatrie und Psychotherapie bzw. zur Fachärztin/ zum Facharzt FMH für Kinder- und Jugendpsychiatrie und -psychotherapie. Die Arbeitsgruppe machte es sich dabei zur Aufgabe, im Sinne der Qualitätssicherung der systemischen Therapie verlässliche Standards zu definieren.

\section{Die «European Family Therapy Association» (EFTA)}

Die EFTA wurde 1992 gegründet. Sie vereinigt in drei Kammern nationale Gesellschaften (Chamber of National Associations), Einzelmitglieder (Chamber of Individual Members) und Ausbildungsinstitute (Chamber of Training and Research Institutes). In über 40 Ländern gehören ihr fast 1000 Einzelmitglieder und 30 Gesellschaften an. Hauptziel der EFTA ist einerseits die Vernetzung und Koordination von nationalen Gesellschaften, Ausbildungsinstituten und Einzelmitgliedern, andererseits die Förderung von Kompetenz und Qualität in systemtherapeutischer Praxis, Forschung, Supervision und Ausbildung.

Die EFTA setzt vier Schwerpunkte:

1. Förderung der Zusammenarbeit und des Austausches zwischen nationalen Gesellschaften, Ausbildungsinstituten und einzelnen Systemtherapeutinnen und -therapeuten;

2. Verbreitung systemtherapeutischen Wissens im psychosozialen Arbeitsfeld;

3. Unterstützung von Weiterbildungsprogrammen für systemtherapeutisch arbeitende Therapeutinnen und Therapeuten;

4. Herstellen von Verbindungen mit anderen therapeutischen Verfahren mit ähnlichen Zielsetzungen.

\section{Fazit}

Die Autoren machen mit ihrem Einsatz für die systemische Therapie auf den Nutzen des Verfahrens für die Gesundheitsversorgung aufmerksam, insbesondere für die krankenkassenärztliche und vertragspsychotherapeutische Versorgung. Wenn man davon ausgeht, 
dass ein Sechstel der Bevölkerung an psychischen Störungen leidet, wobei nur ein Zehntel aller therapiebedürftigen und zugleich auch therapiewilligen Personen tatsächlich ein psychotherapeutisches Angebot erhält, dann kann durch den Einsatz der systemischen Therapie als kurzes und kundennahes Angebot eine Reduktion von Folgekosten von protrahierten psychischen Leidenszuständen und Krankheiten angenommen werden. Wie kontrollierte Studien und Katamnesen zeigen, werden stabile Veränderungen des psychischen Gesundheitszustandes erreicht, indem eine Neugestaltung von Wahrnehmungs-, Handlungs- und Interaktionsmustern angestrebt und realisiert wird. Dies kann einen längeren therapeutischen Prozess erforderlich machen, wie die EAST-Studie [1] gezeigt hat. Die relevanten Bezugspersonen des Patienten werden in die Therapie miteinbezogen. Durch veränderte Interaktionsmuster in Familien und bei Paaren mit einem an psychischen Störungen leidenden Mitglied, durch geänderte Erwartungshaltungen bezüglich der Entwicklung der Krankheit, durch Aktivierung von Ressourcen und Kompetenzen werden Bedingungen geschaffen, um Rückfälle weitgehend zu verhindern. Die systemische Therapie weist ein ausserordentlich breites Indikations- und Anwendungsspektrum auf. Trotz des breiten Spektrums ist damit keine komplizierte und unhandliche Diversifizierung des Verfahrens verbunden. Es handelt sich um ein Verfahren, das nicht nur hinsichtlich der Ergebnisqualität, sondern auch hinsichtlich seiner Prozessqualität positiv zu beurteilen ist. Dies manifestiert sich etwa in einer hohen Klientenzufriedenheit, auf die im Rahmen der Kundenorientierung und des Dienstleistungsgedankens der systemischen Therapie explizit geachtet wird. In die Klientenzufriedenheit gehen Aspekte ein wie Erfolg/ Zielerreichung, Aufwand und Ökonomie des Verfahrens, Erfolgszuschreibungen auf die Eigenleistung des Patienten anstatt auf die Methode. In einer Studie von Preckel und Sauerwald [2] zur systemisch-lösungsorientierten Therapie kam der Berner Klientenstundenbogen (Grawe et al.) [3] zum Einsatz. In der Studie von Nischk und Grothe [4] wurde ein eigener Stundenbogen entwickelt, wobei auch hier die Sitzungen sehr positiv beurteilt wurden. In den Katamnesen von Ludewig [5] äusserten sich zwei Drittel aller Befragten mit den personellen und prozessbezogenen Aspekten der Therapie zufrieden. Der systemische Ansatz ist so konzipiert, dass die für Psychotherapien bekannten möglichen
Nebenwirkungen (z.B. emotionale Dramatik, nachteilige Konsequenzen für die Beziehung zum sozialen Umfeld, Isolation durch die Therapie, Interferenz des therapeutischen Interaktionsstils mit den Kommunikationsstilen der alltäglichen Umwelt, Abhängigkeit von der Therapie) minimiert werden. Die Erfassung von Neben- und Folgewirkungen ist zentraler Bestandteil eines Denkens in Vernetzungen und Systemzusammenhängen und wird im Rahmen von Studien zur Qualitätssicherung der Systemischen Therapie auch realisiert.

Zusammenfassend kann angenommen werden, dass von der systemischen Therapie nicht nur eine qualitative und quantitative Verbesserung der Gesundheitsversorgung der Bevölkerung zu erwarten ist, sondern durch eine Reduktion von Kosten im Gesundheitswesen auch ein volkswirtschaftlicher Vorteil.

Über nationale und internationale Tätigkeiten, Tagungen, Bücher und Diskussionen berichtet regelmässig die Website der Schweizerischen Gesellschaft für Systemische Therapie und Beratung www.sytemis.ch.

Information und Antragsformulare sind erhältlich bei O. Wäger, Präsident der Ärztesektion der SGS, Seemattstrasse 2, 8180 Bülach, Tel. 0186043 20, Fax 0186062 38, E-Mail: ppgwaeger@bluewin.ch, oder dem Kassier der National Family Therapy Organisations (NFTO): E. Louis, 9, route d'Aire-la-Ville, 1233 Bernex, Tel. 02275703 09, Fax 02275703 09, E-Mail: elouis@vtx.ch.

\section{Literatur}

1 Grünwald HS, von Massenbach K. Ergebnisqualität ambulanter systemischer Therapie. Eine Multizenterstudie in der deutschsprachigen Schweiz. Psychother Psych Med 2003;53:326-33.

2 Preckel F, Sauerwald S. Therapeutenverhalten und Klientenerleben. Systematische Unterschiede des Therapeutenverhaltens in von Klienten als hilfreich und nicht hilfreich erlebten lösungsorientierten Kurztherapien. Diplomarbeit, Universität Münster; 1997.

3 Grawe K, Caspar F, Ambühl H. Differentielle Psychotherapieforschung: Vier Therapieformen im Vergleich. Themenheft der Zeitschrift für Klinische Psychologie 1990; 19(4).

4 Nischk D, Grothe A. Welche psychischen Prozesse ermöglichen therapeutische Veränderung? Die empirische Begründung eines neuen Konstrukts: Multidimensionale Veränderungsinvolviertheit. Diplomarbeit, Universität Münster; 1997.

5 Ludewig K. Evaluation systemischer Therapie in einer Kinder- und Jugendpsychiatrie. Erste Ergebnisse. System Familie 1993;6(1):21-35. 